\title{
Predicting sea level variations with artificial neural networks at Hillarys Boat
}

\section{Harbour, Western Australia}

\author{
O. Makarynskyy ${ }^{\mathrm{a},{ }^{*}}$, D. Makarynska ${ }^{\mathrm{b}}$, M. Kuhn ${ }^{\mathrm{a}}$, W.E.Featherstone ${ }^{\mathrm{a}}$ \\ ${ }^{\mathrm{a}}$ Western Australian Centre for Geodesy, Curtin University of Technology, \\ GPO Box U1987, Perth 6845, Australia \\ ' Instituto Superior Técnico, Av.Rovisco Pais, 1, 1049-001 Lisbon, Portugal
}

Keywords: sea level variations; tide gauge; artificial neural networks; forecast; Western Australian coast

\begin{abstract}
In the present study, the artificial intelligence meshless methodology of neural networks was used to predict hourly sea level variations for the following 24 hours, as well as for half-daily, daily, 5-daily and 10-daily mean sea levels. The methodology is site specific; therefore, as an example, the measurements from a single tide gauge at Hillarys Boat Harbour, Western Australia, for the period December 1991 - December 2002 were used to train and to validate the employed neural networks. The results obtained show the feasibility of the neural sea level forecasts in terms of the correlation coefficient (0.7-0.9), root mean square error (about $10 \%$ of tidal range) and scatter index (0.1-0.2).
\end{abstract}

\section{Introduction}

The knowledge of future sea level heights in the nearshore environment is of great importance for protection of coastal and low-lying regions' residents, for monitoring and prediction of changes

\footnotetext{
* Corresponding author.

Tel: +61-89266 7562

Fax: +61-89266 2703

Email: makaryno@vesta.curtin.edu.au (O. Makarynskyy).
} 
in complex marine ecosystems, harvest estimation for the fishery, as well as for planning and constructing coastal and offshore structures, and for the development and implementation of oceanbased alternative energy technologies (e.g., Herbich, 1992; Charlier \& Justus, 1993). In geodesy, national height reference systems are linked to local mean sea level, obtained by long-term measurements at one or more selected tide gauge stations (e.g., Poutanen, 2000). For instance, the Australian Height Datum (AHD) is tied to mean sea level observed over a 2-3 year period at 32 tide gauge stations around Australia (Roelse et al., 1971).

The instantaneous measurements, as well as time-averaged values of sea level, are not stationary either spatially or temporally. They vary under the synergetic influence of changing tides, temperature, salinity, atmospheric forcing, and large-scale ocean currents (e.g., Chen et al., 2000; Douglas, 2000; Ingham, 1992), and sometimes result in storm surges and floods. The transition from deterministic ocean global circulation models with relatively large computational mesh size (tens of kilometres), which among other parameters produce sea level, to local fine scales is a difficult task that demands bulk mathematical apparatus and intensive computations (e.g., Carretero et al., 2000; Monbaliu et al., 2000). The arising problem is especially severe if such a model mesh contains the boundary between the water and land, and consequently large gradients of physical parameters (e.g., Bowden, 1983; Neumann \& Pierson, 1966), which is a common situation in nearshore studies.

To solve the tasks of nearshore sea level predictions, alternative meshless artificial intelligence approaches, like genetic algorithms, fuzzy logic or artificial neural networks, can be employed. For instance, artificial neural networks (ANNs), which are able to approximate any nonlinear mathematical functions (Hornik, 1993), allow plausible simulations of complex systems' behaviour without any preceding knowledge of the internal relations among their components (Haykin, 1999) provided that a reasonably large amount of data has been collected and taken into consideration.

The ANN approach has been successfully used in many studies related to geosciences, such as ocean (Agrawal \& Deo, 2002), coastal (Mase et al., 1995; Tsai et al., 2000; Makarynskyy, 2004), 
environmental (Huang \& Foo, 2002) and land mapping (Mas, 2004) applications. Recently, ANNs were also involved in simulations of sea level variations using the data from the German coastal areas of the North and Baltic Sea (Ultsch \& Roske, 2002), the sea harbours in Taiwan (Lee, 2003), and the Southern Shore of Long Island in the USA (Huang et al., 2003).

The objectives of the current study are to develop and validate a neural methodology applicable to the tasks of site-specific short- and medium-term sea level forecasts. The characteristics of the neural networks, the technique of saliency analysis and the data used in this study are described in Section 2. Sections 3 and 4 discuss the hourly predictions for the next day and the simulations of averaged sea levels at the Hillarys test site, Western Australia, respectively. Some conclusions are presented in Section 5. Importantly, this paper does not mean to cover the environmental impacts of sea level predictions, but instead describe and verify the ANN methodology used.

\section{Techniques and data used}

ANNs are basically parallel information-processing systems. A particular network consists of several neurons, or computational nodes. Taking a weighted input, the node produces the corresponding output by means of an activation, or transfer, function. Several neurons can be combined in a layer. An ANN can contain one or more interconnected layers of neurons where all the neurons are connected to one another. The input layer admits the incoming information, the hidden layer, or layers, processes it, and the output layer presents the network's outcome. The pattern of such interconnections between the nodes in the layers is called the architecture. During the learning process, the weights of the interconnections and the neural biases are iteratively adjusted to minimize the network performance function, which for feed-forward networks is the average squared error between the network outputs and the teaching, or target, outputs. The adjusted weights and biases urge the entire network to perform in some expected way. More 
detailed explanations on artificial neural networks can be found elsewhere (e.g., Zeidenberg, 1990; Fausett, 1994; Bishop, 1995).

Three-layer feed-forward networks were employed in this study, with a non-linear differentiable log-sigmoid transfer function in the hidden layer and linear transfer function in the output layer. The nets were trained with the resilient backpropagation algorithm in 200 training epochs. The feasibility of such networks to geophysical studies was proved by Hornik (1993) and demonstrated in a number of previous works (e.g., Agrawal \& Deo, 2002; Huang \& Foo, 2002; Lee, 2003; Makarynskyy, 2004; Mase et al., 1995; Tsai et al., 2000). An appropriate size of the ANNs was determined applying saliency analysis (Abrahart et al., 2001). Saliency analysis is a technique derived from the idea that an ANN has to stay operational even in the case of incomplete input, or if an internal component faults. The technique allows estimation of the relative importance of the input and processing nodes of a network, as well as its consequent optimization by intentional introduction of missing neurons.

Hourly sea level measurements were obtained from a SEAFRAME (SEA-level Fine Resolution Acoustic Measuring Equipment) station deployed at Hillarys Boat Harbour (Fig.1) at latitude $31.82^{\circ}$ South and longitude $115.73^{\circ}$ East (Geocentric Datum Australia 1994). Since 1991, the equipment has been operated and maintained by National Tidal Centre, Australia, as part of the Australian Baseline Sea Level Monitoring Project in the Australian Greenhouse Science Program. In this study, the whole available period from December 1991 to December 2002 was employed (Fig.2). The raw measurements demonstrate a prominent seasonal variability with annual minima during the Southern Hemisphere summers and maxima in the winters which occur mainly due to the astronomical forcing of the Sun's and Moon's gravitational attractions. The observed values oscillate between $-140 \mathrm{~mm}$ (December 1993) and 1680mm (July 1995) with respect to the unspecified local datum on this decadal time scale, while in a usual year the tidal range does not exceed $1200 \mathrm{~mm}$. This is a relatively mild tidal variation compared to the sites in the north of Western Australia, where the tidal range is of order of several metres. Nevertheless, the study area 
is somewhat challenging because the tide gauge is enclosed in the harbour (Fig.1) that may affect the sea level changes by some internal periodic and/or aperiodic resonant oscillations, but these lie beyond the scope of this case study. Therefore, the results obtained here may not necessarily be generalized for other locations on the Western Australian coast, where independent site-specific studies should be performed.

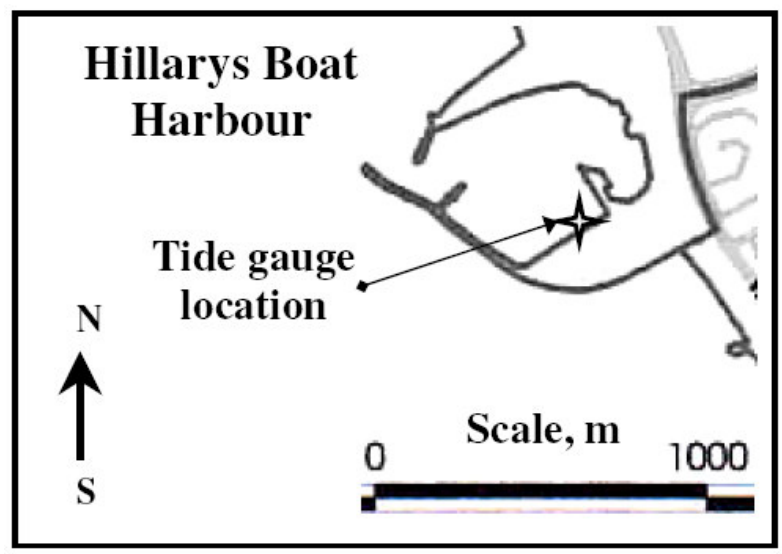

Fig. 1. Location of the SEAFRAME tide gauge (star) at Hillarys Boat Harbour

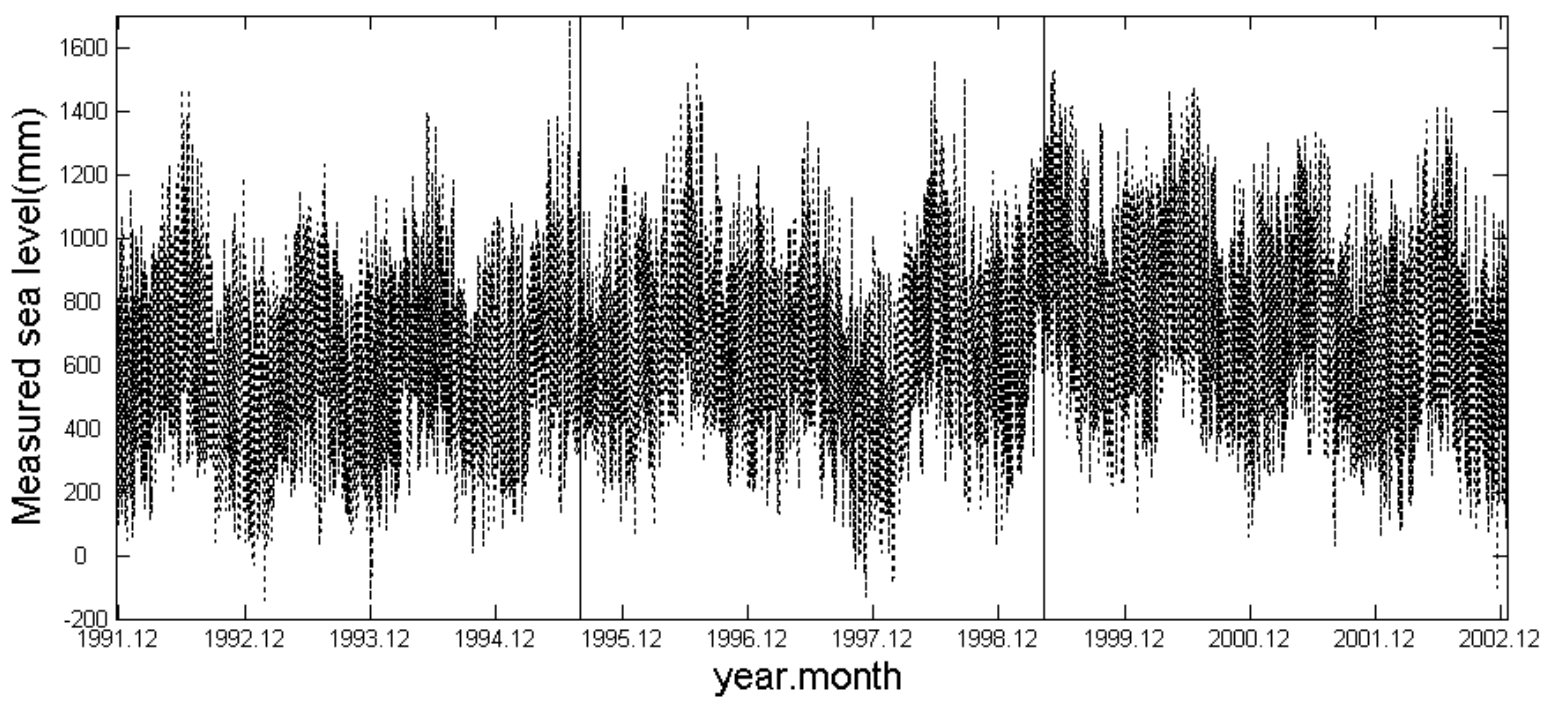

Fig. 2. Hourly sea level observations from the Hillarys Boat Harbour SEAFRAME tide gauge.

Three time series for independent training of the forecasting (first part), correcting (second part) ANNs, and for general validation (third part) are separated by vertical solid lines 


\section{Hourly sea level forecasts}

\subsection{Predictions}

In the first set of experiments, the measurements from the Hillarys tide gauge were divided into three equal-length data sets of 32324 sea level observations. The first part of the observations (December 1991-September 1995) was used to train the forecasting ANNs; the second part (September 1995-May 1999) was used for independent training of the correcting networks, while the last portion (May 1999-December 2002) served for validation of both (Fig. 2). The performance was evaluated in terms of the correlation coefficient $R$, root mean square error $R M S E$, and scatter index $S I$, computed as

$$
\begin{aligned}
& R=\frac{\sum_{i=1}^{N}\left(x_{i}-\bar{x}\right)\left(y_{i}-\bar{y}\right)}{\sqrt{\sum_{i=1}^{N}\left(x_{i}-\bar{x}\right)^{2} \sum_{i=1}^{N}\left(y_{i}-\bar{y}\right)^{2}}}, \\
& R M S E=\sqrt{\frac{\sum_{i=1}^{N}\left(y_{i}-x_{i}\right)^{2}}{N}}, \\
& S I=\frac{R M S E}{\bar{x}},
\end{aligned}
$$

where $x_{i}$ is the value observed at the $i$-th time step, $y_{i}$ is the value simulated at the same moment of time, $N$ is the number of time steps, $\bar{x}$ is the mean value of the observations, and $\bar{y}$ is the mean value of the simulations.

Using the saliency analysis technique (Section 2), ANNs with architectures ranging from 72 input neurons -145 processing neurons - 24 output neurons (hereafter referred to as 72 x $145 \times 24$, and likewise for other architectures) to $12 \times 25 \times 24$ were explored to produce one-day (24 hours) forecasts of hourly sea level variations (Table 1). The quasi-diurnal (K1, O1 and P1) and quasisemidiurnal (M2) tide waves are the dominant ones in the area of Hillarys Boat Harbour (e.g., 
Australian National Tide Tables 2003). Therefore, taking the semidiurnal and diurnal periodicities into account in the learning phase, the measurements were fed into the predicting networks in 12hour increments. Thus, during one training epoch, the input-output pattern presented, for instance, to the network $72 \times 145 \times 24$ was as follows. In the first learning step, the observations from hours 1 to 72 served as the input information, while the sea levels from hours 73-96 were introduced to the network as the target values. In the second learning step, the measurements from hours 13-84 were the input and from hours 85-108 were the target, and so on. The learning process continued until all the data in the training subseries were used.

Table 1. Number of nodes in the input and hidden layers of the forecasting ANNs. The presented statistics were averaged over the 24-hour interval of forecast

\begin{tabular}{ccccc}
\hline Input & Hidden & Averaged & Averaged & Averaged \\
neurons & neurons & $R$ & $R M S E(\mathrm{~mm})$ & $S I$ \\
\hline 72 & 145 & 0.816 & 139 & 0.195 \\
\hline 60 & 121 & 0.830 & 130 & 0.182 \\
\hline 48 & 97 & 0.859 & 118 & 0.165 \\
\hline 36 & 73 & 0.859 & 119 & 0.166 \\
\hline 24 & 49 & 0.875 & 111 & 0.155 \\
\hline 12 & 25 & 0.823 & 122 & 0.172
\end{tabular}

Figure 3 displays time-series plots of the hourly sea level forecast statistics and ensemble average values. The latter are the mean of the statistical characteristics obtained over each lead-time from 1 hour to 24 hours. From an analysis of Fig. 3 and Table 1, it follows that, in general, all the networks perform in a similar way. However, the two networks with the largest numbers of neurons in the input and hidden layers (i.e., $72 \times 145 \times 24$ and $60 \times 121 \times 24$ ) produce predictions with consistently lower accuracy than the three neural nets of simplified architecture (48 x $97 \times 24,36 \times$ 
$73 \times 24$ and $24 \times 49 \times 24)$. The overall best performance was attained with the network with an even number of input-output nodes, and a number of hidden units, or processing neurons, equal to (input nodes + output nodes $)+1$ (see also Huang \& Foo, 2002). That is, the net with 24 input units, 49 processing neurons, 24 output nodes. The averaged statistics over all 24 predicting intervals confirm the same trends (Table 1). This means that, for this case study, too much initial information and an overcomplicated network architecture deteriorate the quality of simulations because the more complex networks are not able to detect any consistent relations among so many input-processingoutput components. This, in turn, underlines the suitability of saliency analysis for the improvement of sea level forecast in terms of resulting accuracy. The decrease of the number of neurons also leads to smaller computational efforts.

From Fig. 3 and Table 1, the network with the fewest number of input and processing units simulates sea levels slightly better than the others tested for time intervals of 1-2 and 14-24 hours ahead, and worse in the forecasts for 5-10 hours. The latter clearly affects the ensemble and the network's time averages. Therefore, as the main interest lies in the overall quality of one-day hourly predictions, it was inferred that too few initial data (12 hourly measurements for this case) are not sufficient for an accurate simulation of the sea level variability for the next 24 hours. This is plausible given the typical tidal periods.

Another noteworthy feature present in Fig. 3 is an abrupt decrease in accuracy between the fifth and tenth hours of the forecasts. This is attributed to the use of 12-hour training increments. Reducing the increments to 6 or 3 hours, or to one hour, would assist in revealing consistent patterns disguised in the measurements. However, some negative effects itemized below of a finer partition may manifest themselves. Firstly, refining time increments will serve more training patterns demanding more computational resources without providing any additional new data to the ANNs. Secondly, an enhanced number of training pairs may eventually lead to the problem of overfitting, or overlearning, when an ANN extracts too much information from some individual cases and becomes unable to reproduce important general features. 

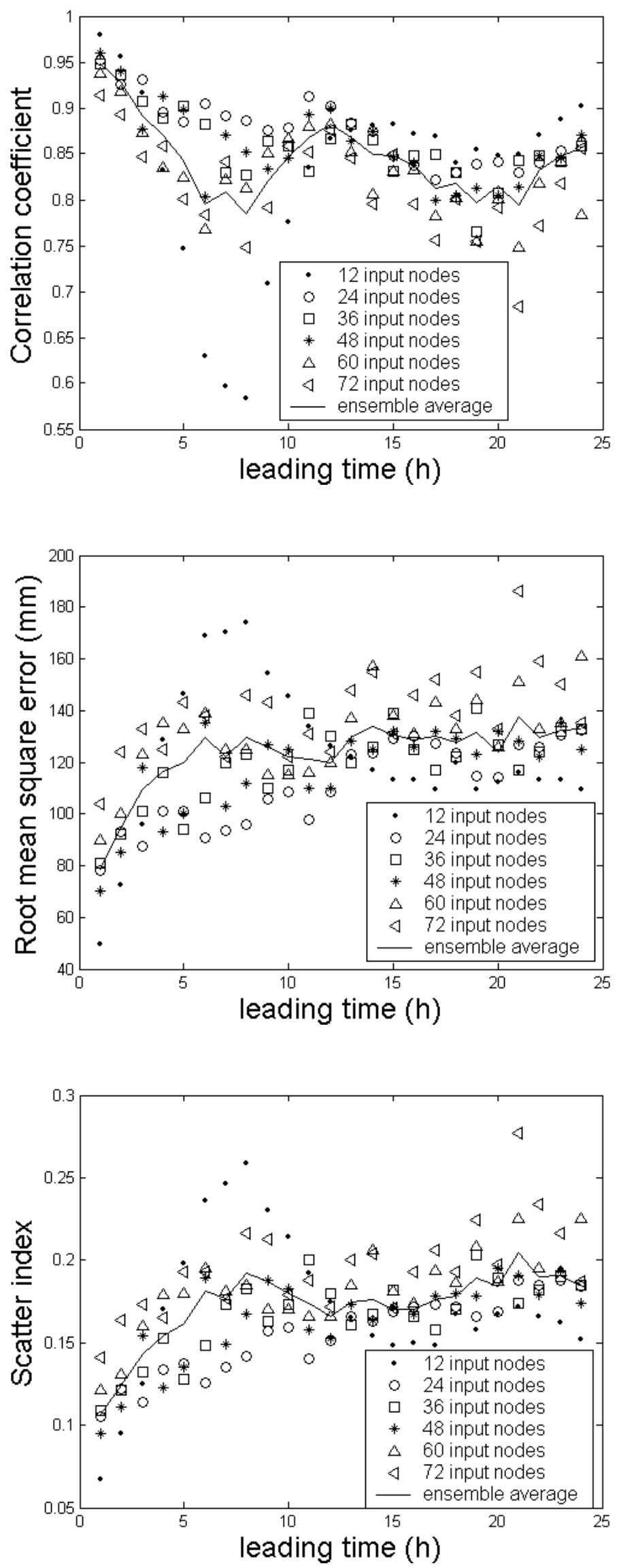
Fig. 3. Correlation coefficients $(R)$, root mean square errors $(R M S E)$ and scatter indexes $(S I)$ for each leading time of initial simulations

\subsection{Correcting predictions}

The use of saliency analysis allowed partial improvement of the methodology's performance. Assuming that a network of different internal structure might be trained for reproducing a more plausible output, an additional neural procedure was developed in attempt to correct the initial sea level simulations. The ancillary nets used had 24 nodes in both the input and output layers according to the number of the prediction intervals in the initial and final simulations, and 49 neurons in the hidden layer. These correcting nets were trained as described in Section 3.1.

A scrutiny of Fig. 4 results in the following observations. The differences in correlation coefficients of the initial ( $48 \times 97 \times 24$ and $36 \times 73 \times 24$ networks) and corrected predictions are mostly negative, while the differences in the RMSE and SI are generally positive. These indicate that the quality of the initial simulations was improved by employing the supplementary neural procedure. Scatter diagrams "simulations versus measurements" (Fig.5) illustrate the improvement, showing a generally higher degree of spreading of the initial forecasts around the exact fit line compared to the corrected predictions.

Notably, fairly accurate initial simulations produced by the 24 x 49 x 24 network (Table 1) were not improved at all. This is attributed to the fact that the correction procedure merely adds to the total number of training epochs of the net with the same architecture, thus leading to the negative effect of overlearning. 

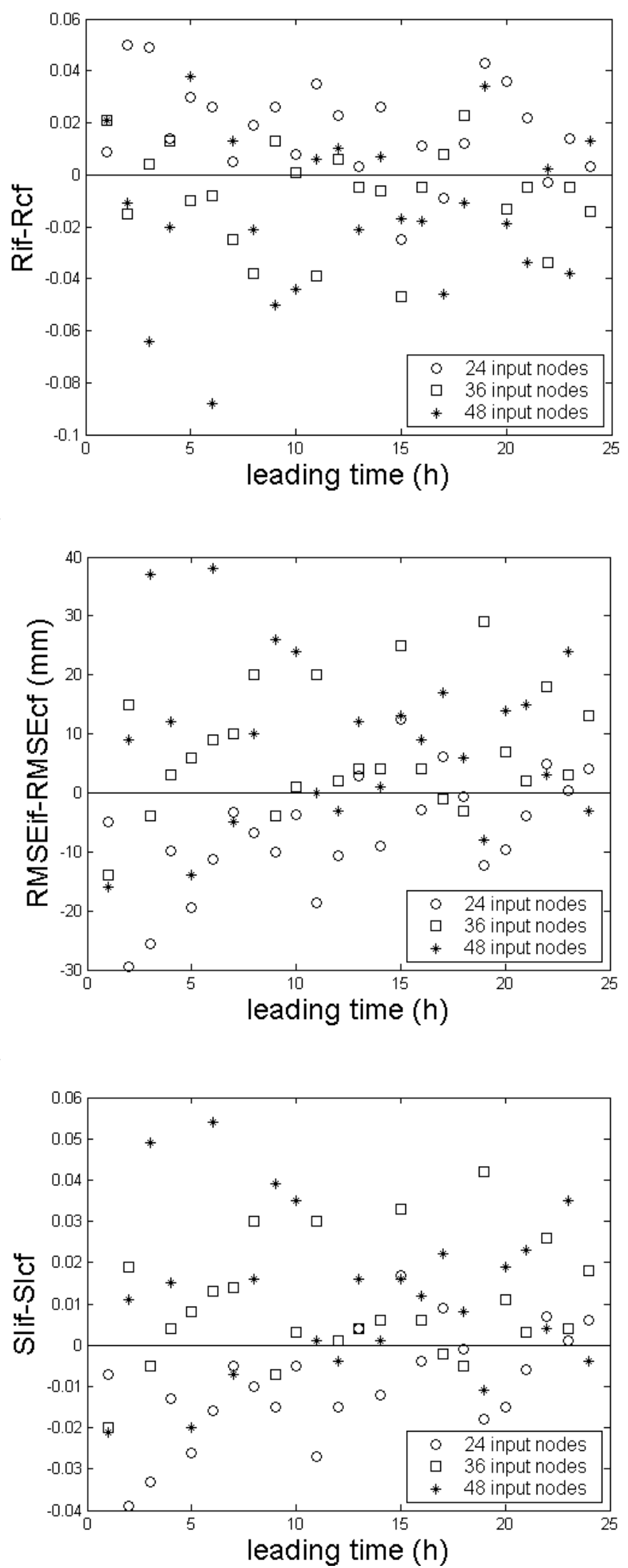

Fig. 4. Differences between correlation coefficients $(R)$, root mean square errors $(R M S E)$ and scatter indexes (SI) of the hourly initial forecasts (Rif, RMSEif and Slif, respectively) by three different neural networks and corrected forecasts (Rcf, RMSEcf and SIcf, respectively) 

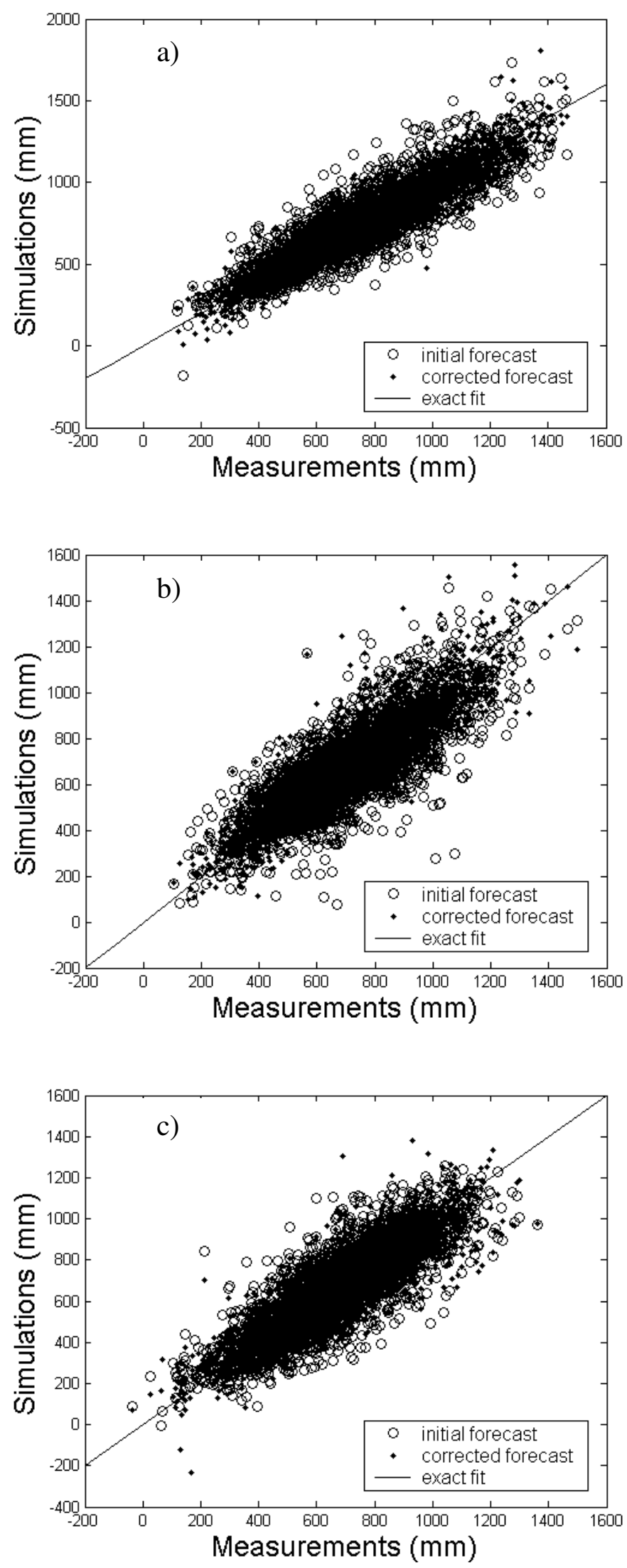

Fig. 5. Scatter plot of simulations 3 hours (a), 6 hours (b) and 9 hours (c) ahead versus observations and the line of exact fit. Predicting network 48x97x24 


\section{Simulations of average sea levels}

To accomplish the second imposed task of mid-term forecast of mean sea level, the initial data were averaged over time intervals of 12, 24, 120 and 240 hours (Fig. 6). Thus, compared to the previous set of experiments, the resulting numbers of the available input-output pairs were significantly reduced. To provide quality training on fewer numbers of patterns, it was decided to skip the correction procedure in this case and to use three-quarters of the series (December 1991March 1999) for training purposes, and the rest of the data (March 1999-December 2002) for an independent validation (see Eqs.1-3) of the ANNs used.

Based on the previous findings, the neural networks with even number of input and output neurons, and a number of processing units $=($ input nodes + output nodes $)+1$ were employed . The learning algorithm and training epochs used were the same as in Section 3.1. Saliency analysis of the predictions obtained applying the networks $10 \times 21 \times 10,8 \times 17 \times 8,6 \times 13 \times 6,4 \times 9 \times 4$ (not shown) and $3 \times 7 \times 3$ (Table 2) exhibited a higher accuracy of the net with the simplest architecture, independent of the averaging interval. Hence, four different ANNs of the same architecture spanning three previous averaged values were used to predict the mean sea level up to three steps (time intervals) ahead.

An analysis of the statistics in Table 2 demonstrates that, in general, mean sea levels were simulated reasonably well with low values of the RMSE and SI and high $R$. Especially successful were the predictions for the first two time steps. It is clear that 12-hour and 24-hour averaged sea levels provide a reliable basis for 12-hour and one-day-ahead forecasts. However, this was not the case when longer-term predictions for 36,48 and 72 hours were concerned. Respectively, the quality of simulations of 5-day and 10-day averages was reasonably high over time intervals of 5, 10 and 20 days, with less success in 15- and 30-day predictions. The lower accuracy of the "third time step" simulations might be attributed to the reduced number of training patterns as well as to weak interdependencies between the values used as input-target pairs. 

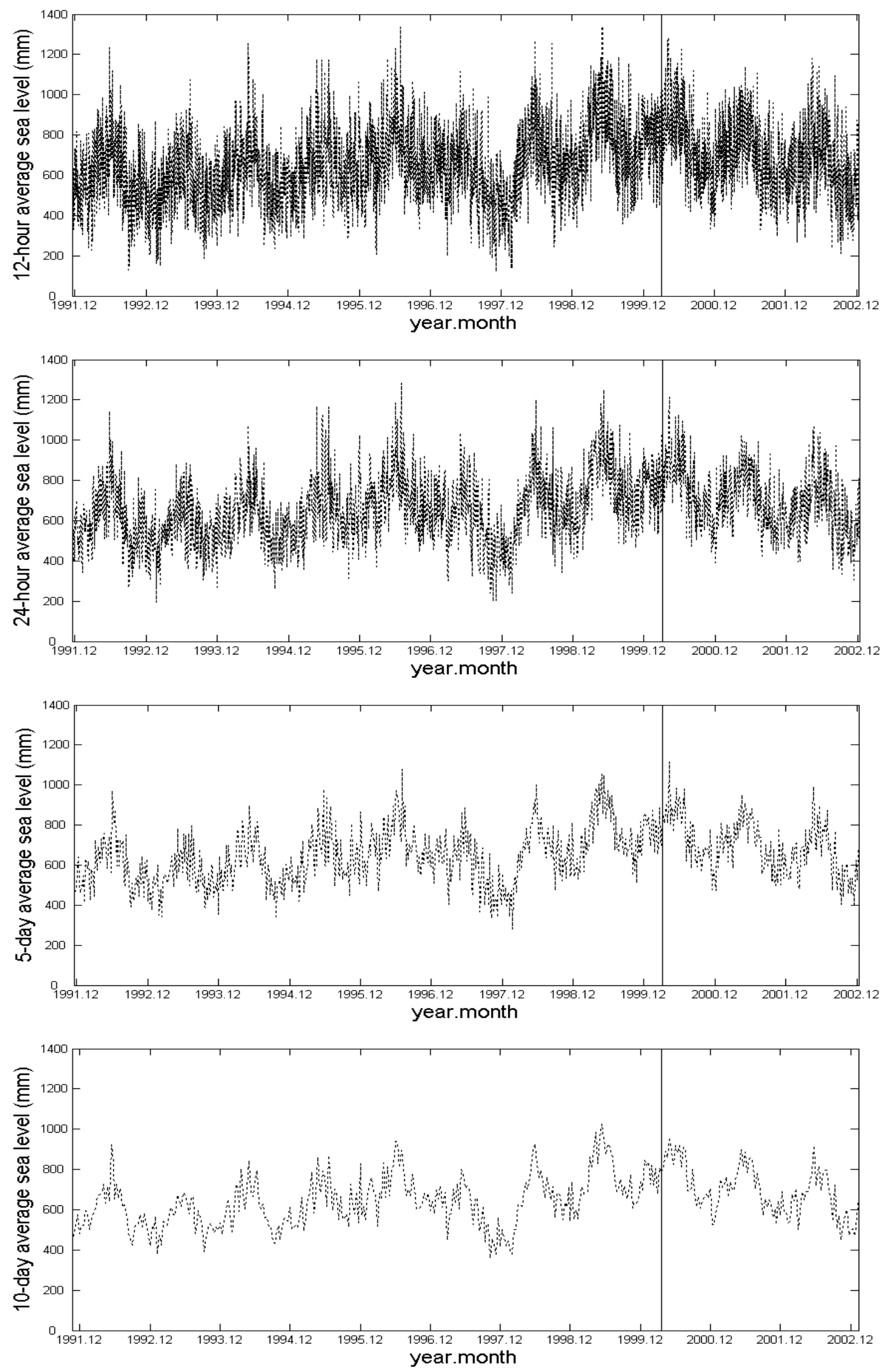

Fig. 6. Mean sea levels averaged over 12- and 24-hour, and 5- and 10-day periods. Time series used for training and validation are separated by vertical solid line 
Table 2. Statistics of the simulations of 12-hour, 24-hour, 5-day and 10-day averages

\begin{tabular}{|c|c|c|c|c|c|c|c|}
\hline \multirow{2}{*}{$\begin{array}{l}\text { Prediction } \\
\text { interval }\end{array}$} & \multicolumn{3}{|c|}{ 12-hour average } & \multirow{2}{*}{$\begin{array}{l}\text { Prediction } \\
\text { interval }\end{array}$} & \multicolumn{3}{|c|}{ 24-hour average } \\
\hline & $\mathrm{R}$ & RMSE (mm) & SI & & $\mathrm{R}$ & RMSE (mm) & SI \\
\hline+12 hours & 0.894 & 115 & 0.164 & +24 hours & 0.872 & 73 & 0.105 \\
\hline+24 hours & 0.859 & 87 & 0.125 & +48 hours & 0.667 & 115 & 0.165 \\
\hline+36 hours & 0.679 & 186 & 0.267 & +72 hours & 0.558 & 69 & 0.099 \\
\hline Prediction & & 5-day average & & Prediction & & 10-day average & \\
\hline interval & $\mathrm{R}$ & RMSE (mm) & SI & interval & $\mathrm{R}$ & RMSE (mm) & SI \\
\hline+5 days & 0.722 & 81 & 0.114 & +10 days & 0.785 & 71 & 0.101 \\
\hline+10 days & 0.700 & 80 & 0.116 & +20 days & 0.736 & 90 & 0.129 \\
\hline +15 days & 0.669 & 87 & 0.124 & +30 days & 0.667 & 151 & 0.216 \\
\hline
\end{tabular}

\section{Conclusions}

The sea level observations from the Hillarys Boat Harbour tide gauge on the coast of Western Australia were used for development and validation of an ANN methodology for sea level forecasts. The commonly used three-layer feed-forward networks with a non-linear differentiable log-sigmoid transfer function in the hidden layer and linear transfer function in the output layer were employed in this case study. In general, satisfactory predictions with the values of correlation coefficients equal to $0.7-0.9$, root mean square errors of about $10 \%$ of the tidal range and scatter indexes equal to $0.1-0.2$ were produced. The validated neural methodology can be successfully applied to other coastal regions provided site-specific trainings and validations are carried out appropriately.

In the first set of simulations, the neural technique was successfully implemented to predict hourly sea levels with leading times from 1 to 24 hours and, further, to correct the results of the initial simulations. An ancillary correcting neural network improved the quality of the initial hourly 
simulations when assimilating the outcomes of more complex nets, while its application led to overfitting when the input data were coming from the network with the same number of inputprocessing-output units.

The usefulness of saliency analysis was highlighted. This technique helps in optimal network architecture selection that may consequently lead to better performance of the net and smaller computational efforts needed to train it.

In the second set of simulations, ANNs were implemented to forecast sea levels averaged over 12-hour, 24-hour, 5-day and 10-day time periods, three time steps ahead. This produced highquality predictions over first two time steps rather than over the third time interval. The lack of accuracy in the latter was attributed to the reduced number of the input-output training pairs and weak interrelations among them.

\section{Acknowledgments}

This study was partially funded by the ARC Discovery-Project grant DP0345583 "Prediction of Sea Level Change around Australia and its Calibration and Validation by Satellite-Geodetic Measurements". The authors are grateful to the National Tidal Centre of Australia for making available the tide gauge observations. Part of this work was done when Dina Makarynska was a doctoral fellow at the "Fundação para a Ciência e Tecnologia" of the Ministry of Science and High Education of Portugal. The authors also thank the reviewers for their constructive comments.

\section{References}

Abrahart, R.J., See, L., Kneal, P.E., 2001. Investigating the role of saliency analysis with neural network rainfall-runoff model. Computers \& Geosciences, 27, 921-928, doi:10.1016/S00983004(00)00131-X. 
Agrawal, J.D., Deo, M.C., 2002. On-line wave prediction. Marine Structures, 15, 57-74, doi:10.1016/S0951-8339(01)00014-4.

Australian National Tide Tables 2003. Australian Hydrographic Publication 11, Department of Defence. 404pp.

Bishop, C.M., 1995. Neural Networks for Pattern Recognition. Oxford University Press, 504 pp.

Bowden, K. F., 1983. Physical Oceanography of Coastal Waters. Halsted Press, 302pp.

Carretero, J. C., Alvarez, E., Gomez, M., Perez, B., Rodríguez, I., 2000. Ocean forecasting in narrow shelf seas: application to the Spanish coasts. Coastal Engineering, 41 (1-3), pp. 269-293, doi:10.1016/S0378-3839(00)00035-1.

Charlier, R.H., Justus, J.R., 1993. Ocean energies. Elsevier, 554pp.

Chen, J.L., Shum, C.K., Wilson, C.R., Chambers, D.P., Tapley, B.D., 2000. Seasonal sea level change from TOPEX/Poseidon observation and thermal contribution. Journal of Geodesy, 73, 638-647.

Douglas, B.C., Kearney, M.S., Leatherman, S.P., 2000. Sea Level Rise History and Consequences, International Geophysics Series, Academic Press London, 75, 97-119.

Fausett, L., 1994. Fundamentals of neural networks. Architectures, algorithms, and applications. Prentice-Hall, Upper Saddle River, NJ, 462 p.

Haykin, S., 1999. Neural networks: a comprehensive foundation. Prentice-Hall, Upper Saddle River, NJ, 842pp.

Herbich, J.B., 1992. Handbook of coastal and ocean engineering. Gulf Professional Publishing, 1340pp.

Hornik, K., 1993. Some new results on neural network approximation. Neural Networks, 6, 10691072.

Huang, W., Foo, S., 2002. Neural Network Modelling of Salinity Variation in Apalachicola River. Water Research, 36, 356-362, doi:10.1016/S0043-1354(01)00195-6. 
Huang, W., Murray, C., Kraus, N., Rosati, J., 2003. Development of a regional neural network for coastal water level predictions. Ocean Engineering, 30, 2275-2295, doi:10.1016/S00298018(03)00083-0.

Ingham, A.E., 1992. Hydrography for the surveyor and engineer. Blackwell Science UK, 132pp.

Lee, T.L., 2004. Back-propagation neural network for long-term tidal predictions, Ocean Engineering, 31 (2), 225-238, doi:10.1016/S0029-8018(03)00115-X.

Makarynskyy, O., 2004. Improving wave predictions with artificial neural networks, Ocean Engineering, 31 (5-6), 709-724, doi:10.1016/j.oceaneng.2003.05.003.

Mas, J. F., 2004. Mapping land use/cover in a tropical coastal area using satellite sensor data, GIS and artificial neural networks. Estuarine, Coastal and Shelf Science, 59 (2), 219-230, doi:10.1016/ j.ecss.2003.08.011.

Mase, H., Sakamoto, M., Sakai, T., 1995. Neural network for stability analysis of rubble-mound breakwaters, J. Waterway, Port, Coastal and Ocean Engineering., ASCE, 121 (6), 294-299.

Monbaliu, J., Padilla-Hernández, R., Hargreaves, J.C., Carretero Albiach, J.C., Luo, W., Sclavo, M., Günther, H., 2000. The spectral wave model, WAM, adapted for applications with high spatial resolution. Coastal Engineering, 41 (1-3), 41-62, doi:10.1016/S0378-3839(00)00026-0.

Neumann, G., Pierson, W.J., 1966. Principles of physical oceanography. Prentice-Hall. Englewood Cliffs. 545 pp.

Poutanen, M., 2000. Sea surface topography and vertical datums using space geodetic techniques. Publications of the Finnish Geodetic Institute, N.128, Kirkkonummi, 312pp.

Roelse, A., Granger, H.W., Graham, J.W., 1971. The adjustment of the Australian levelling survey - 1970-71. Technical Report 12, Division of National Mapping, Canberra.

Tsai, C.P., Hsu, J.R.-C., Pan, K.L, 2000. Prediction of storm-built beach profile parameters using neural network, Proc.27th Int. Conf. Coastal Engineering, ASCE, V.4, 3048-3061.

Ultsch, A., Roske, F., 2002. Self-organizing feature maps predicting sea levels, Information Sciences, 144, 91-125, doi:10.1016/S0020-0255(02)00203-7. 
Zeidenberg, M., 1990. Neural network models in artificial intelligence. Prentice Hall, Ellis Horwood, Chichester, 268pp. 